Pacific Journal of Mathematics

Corrosion RADICALS 


\section{ON COTORSION RADICALS}

\section{S. Ramamurthi and E. A. Rutter, Jr.}

Necessary and sufficient conditions are obtained for the splitting of a hereditary cotorsion radical on the category $\mathcal{M}_{R}$ of right $R$-modules. It is shown that for injective cogenerator rings and orders in semi-simple rings, all hereditary cotorsion radicals split. Several other questions are also discussed. These include the relationship between cotorsion radicals for $\mathcal{M}_{R}$ and ${ }_{R} \mathcal{M}$, and the connection between the properties of a projective module and those of the cotorsion radical it induces.

Introduction. The notion of a cotorsion radical for the category $\mathcal{M}_{R}$ of right modules over a ring $R$ was introduced by Beachy [3], as the dual to the notion of a torsion radical due to Maranda [8]. A cotorsion radical for $\mathcal{M}_{R}$ is a subfunctor $\rho$ of the identity functor on $\mathcal{M}_{R}$ such that $\rho^{2}=\rho$ and every epimorphism $M \rightarrow N$ in $\mathcal{M}_{R}$ induces an epimorphism $\rho(M) \rightarrow \rho(N)$. If $\rho$ is a cotorsion radical, then the class of modules $\mathcal{M}$ for which $\rho(M)=0$ is a torsion radical class; so the study of cotorsion radicals yields information which is useful in studying TTF theories (e.g. see Jans [6] and Tepley [12]).

A cotorsion radical $\rho$ is called hereditary if it is left exact and splitting if $\rho(M)$ is a direct summand of $M$ for every $M$ in $\mathcal{M}_{R}$. This paper continues the investigation of the heredity and splitting of cotorsion radicals begun in [10]. The quotient ring of any ring $R$ with respect to any hereditary cotorsion radical on $\mathcal{M}_{R}$ is determined. A necessary and sufficient condition for a hereditary cotorsion radical to split is given and it is shown that for injective cogenerator rings and orders in semisimple rings, all hereditary cotorsion radicals split. The relationship between the cotorsion radicals for $\mathcal{M}_{R}$ and those for ${ }_{R} \mathcal{M}$ is discussed in the second section. A one-to-one correspondence which preserves splitting is shown to exist between the hereditary cotorsion radicals for $\mathcal{M}_{R}$ and those for ${ }_{R} \mathcal{M}$. The final section studies the cotorsion radicals induced by projective modules. The question of heredity of such radicals is shown to be related to the concepts of perfect injectors and perfect projectors introduced by Anderson in [1]. Finally some connections between flat modules and torsion radicals are studied.

Terminology. All rings considered here have identities and all modules are unitary. Ideal means a two-sided ideal. The injective hull of a module $M$ will be denoted by $E(M)$. A ring $R$ is called a left (respectively right) 1-FGFP ring, if each cyclic flat left (respectively right) 
$R$-module is projective. This class of rings is useful because it includes several well-known types of rings. For instance Noetherian rings and semiperfect rings are 1-FGFP rings as are orders in semi-simple Artinian rings. This list can easily be lengthened because subrings of 1-FGFP rings are 1-FGFP rings [Jondrup [7]]. $R$ is called a right injective cogenerator ring, if $R_{R}$ is injective and every right $R$-module is a submodule of a direct product of copies of $R_{R}$. A semiperfect ring (respectively left perfect ring) is a ring all of whose finitely generated right modules (respectively all of whose right modules) have projective covers. A ring is said to be primary decomposable if it is a finite direct sum of left perfect rings each of which has a unique maximal two-sided ideal.

1. Hereditary cotorsion radicals. A subfunctor $\rho$ of the identity functor on $\mathcal{M}_{R}$ is called a torsion radical if $\rho$ is left exact and $\rho(M / \rho(M))=0$ for each $M$ is $\mathcal{M}_{R}$ [Maranda [8]]. $\rho$ is called a cotorsion radical, if the dual of the functor $1 / \rho: M \rightarrow M / \rho(M)$ on $\mathcal{M}_{R}$, in the dual category $\mathcal{M}_{R}^{*}$ is a torsion radical on $\mathcal{M}_{R}^{*}$ [Beachy [3]]. Beachy has shown that, for any cotorsion radical $\rho$ on $\mathcal{M}_{R}, \rho(M)=M \cdot \rho(R)$ for every $M$ in $M_{R}$ and that the correspondence $\rho \rightarrow \rho(R)$ is onerone between the cotorsion radicals on $\mathcal{M}_{R}$ and the idempotent ideals of $R$. It follows from [3, Prop. 1.1] that a cotorsion radical is a torsion radical if and only if it is left exact or equivalently $\rho(M)=M \cap \rho(N)$ for any two modules $M, N \in M_{R}$ with $M \subset N$. Cotorsion radicals with the latter property have been called hereditary in [Ramamurthi [10]] and the following characterization obtained.

Proposition 1.1. A cotorsion radical $\rho$ on $\mathcal{M}_{R}$ is hereditary if and only if $R / \rho(R)$ is left $R$-flat.

Let $\rho$ be a torsion radical. For each right $R$-module $M$ define the module of quotients $M_{\rho}$ of $M$ with respect to $\rho$ by $M_{\rho} / \bar{M}=\rho[E(\bar{M}) / \bar{M}]$ where $\bar{M}=M / \rho(M)$. Then $R_{\rho}$ can be given a unique ring structure which extends the ring structure of $\bar{R}$ and is called the ring of quotients of $R$ with respect to $\rho$. Similarly, there is induced a unique $R_{\rho}$-module structure on $M_{\rho}$ that extends its $R$-module structure. This process defines a functor from $\mathcal{M}_{R}$ to $\mathcal{M}_{R_{p}}$. When this functor is naturally equivalent to ( $) \otimes_{R} R_{\rho}, \rho$ is called a perfect torsion radical. If $\rho$ is a hereditary cotorsion radical, then as mentioned above, $\rho$ is also a torsion radical. The next proposition shows that $\rho$ is in fact a perfect torsion radical and the quotient functor it determines is just ()$\bigotimes_{R} R / \rho(R)$.

Proposition 1.2. Let $\rho$ be a hereditary cotorsion radical for $M_{R}$. For each $M$ in $M_{R}$, the module of quotients of $M$ with respect to $\rho$ is $M / M \rho(R)$. 
Proof. Let $\bar{M}=M / \rho(M)=M / M \rho(R)$ and $X=\rho(E(\bar{M})) \cap \bar{M}$. Then $\rho(X)=X$ since $X \subset \rho(E(\bar{M}))$ and $\rho$ is hereditary. But $\rho(X)=0$ since $X \subset \bar{M}$ and $\rho(\bar{M})=0$. Thus $X=0$ which means $\rho(E(\bar{M}))=0$ as $E(\bar{M})$ is an essential extension of $\bar{M}$. Hence $\rho[E(\bar{M}) / \bar{M}]=0$ because $\rho$ is a cotorsion radical. Thus $M_{\rho}=\bar{M}$.

Let us say that a cotorsion radical $\rho$ for $\mathcal{M}_{R}$ commutes with direct products, if $\rho\left(\Pi M_{\alpha}\right)=\Pi \rho\left(M_{\alpha}\right)$ for each family $\left\{M_{\alpha}\right\}$ of right $R$-modules.

Proposition 1.3. A cotorsion radical $\rho$ commutes with direct products if and only if $\rho(R)$ is finitely generated as a left ideal.

Proof. Assume $\rho$ commutes with direct products. Let $I=\rho(R)$ and consider $\Pi_{a \in I} R_{a}$ where each $R_{a}=R$. Since $\rho\left(\Pi R_{a}\right)=\Pi \rho\left(R_{a}\right)$, we have (II $\left.R_{a}\right) \cdot I=\Pi I_{a}$ where $I_{a}=I$ for all $a \in I$. Hence, the element $\left\{x_{a}\right\}_{a \in I}$ with $x_{a}=a$ for all $a \in I$, belongs to $\left(\Pi R_{a}\right) \cdot I$ and so is of the form $\sum_{i=1}^{k} r_{i} b_{i}$ for some $r_{i} \in \Pi R_{a}$ and $b_{\imath} \in I$. It is clear that the $b_{\imath}$ generate $I$ as a left ideal. The converse verification is routine.

The next corollary has been obtained, independently, by Azumaya [2, Theorem 3] who phrased it in terms of TTF classes.

COROLlaRY 1.4. A cotorsion radical $\rho$ is hereditary and commutes with direct products if and only if $\rho(R)=\operatorname{Re}$ for an idempotent $e$.

Proof. Follows from (1.3) and (1.1) since finitely presented flat modules are projective [5].

A cotorsion radical $\rho$ is said to split a module $M$ in $\mathcal{M}_{R}$ if $\rho(M)$ is a direct summand of $M$. If $\rho$ splits all $M$ in $\mathcal{M}_{R}$, then $\rho$ is called a splitting cotorsion radical.

THEOREM 1.5. The following are equivalent on any hereditary cotorsion radical $\rho$ on $\mathcal{M}_{R}$.

(1) $\rho$ is a splitting cotorsion radical.

(2) $\rho$ splits all injective modules in $M_{R}$.

(3) $\rho$ commutes with direct products and $\rho(E(S))^{\prime}=0$ or $E(S)$ for all simple modules $S$ in $M_{R}$.

(4) $\rho(R)=\operatorname{Re}$ for a central idempotent e of $R$.

Proof. $1 \Rightarrow 4$ : Let $R=\rho(R) \oplus K$ where $K$ is a right ideal of $R$. Clearly $R=\rho(R)+R K$. Since $\rho$ is hereditary, $\rho(R) \cap R K=$ $\rho(R K)=R K \cdot \rho(R)$. Thus $\rho(R)$ is a ring direct summand of $R$ and so equals $\operatorname{Re}$ for a central idempotent $e$ of $R$.

$4 \Rightarrow 3$ : The first part follows by (1.3). To prove the second part, let $S$ be a simple module in $M_{R}$ such that $\rho(E(S))=E(S) \cdot e \neq 0$. Then 
$E(S)=E(s) \cdot e \bigoplus E(S) \cdot(1-e)$. This implies that $E(S) \cdot(1-e)=0$ as $E(S)$ is an essential extension of $S$. Thus $E(S)=E(S) \cdot e=\rho(E(S))$.

$3 \Rightarrow 2$ : If $M$ is an injective in $M_{R}$, then $E(\rho(M)) \subset M$. Thus $\rho(E(\rho(M)))=\rho(M)$ as $\rho$ is hereditary. But $M \subset \Pi E\left(S_{\alpha}\right)$ for a family of simple modules $\left\{S_{\alpha}\right\}_{\alpha \in I}$. So $\rho(M) \subset \rho\left[\Pi E\left(S_{\alpha}\right)\right]=\Pi \rho\left(E\left(S_{\alpha}\right)\right) \simeq$ $\Pi_{\beta \in J} E\left(S_{\beta}\right)=N$ where $J=\left\{\alpha \in I ; \rho\left(E\left(S_{\alpha}\right)\right)=E\left(S_{\alpha}\right)\right\}$. Since $N$ is injective, $E(\rho(M)) \subset N$ and further $\rho(N)=N$. Hence $\rho(E(\rho(M)))=$ $E(\rho(M))$. Thus $\rho\left(M^{*}\right)=E(\rho(M))$ and so $\rho$ splits $M$.

$2 \Rightarrow 1$ : First note that the hypothesis is equivalent to: $\rho(M)=M$ implies $\rho(E(M))=E(M)$ for any $M$ in $\mathcal{M}_{R}$. Now, let $\left\{M_{\alpha}\right\}$ be a family of right $R$-modules with $\rho\left(M_{\alpha}\right)=M_{\alpha}$ for each $\alpha$. Then, as $\rho$ is hereditary, $\rho\left(\Pi M_{\alpha}\right)=\left(\Pi M_{\alpha}\right) \cdot \rho(R)$ is an essential submodule of $\Pi M_{\alpha}$ and hence $\Pi M_{\alpha} \subset E\left(\rho\left(\Pi M_{\alpha}\right)\right)$. But, by hypothesis, $\rho\left(E\left(\rho\left(\Pi M_{\alpha}\right)\right)\right)=$ $E\left(\rho\left(\Pi M_{\alpha}\right)\right)$ so that $\rho\left(\Pi M_{\alpha}\right)=\Pi M_{\alpha}$. Thus $\rho$ commutes with direct products and hence, by (1.4), $D=\rho(R)=\operatorname{Re}$ for an idempotent $e$. Let $K=(1-e) R$ and consider $\rho(K)$. The inclusion morphism $\rho(K) \rightarrow E(\rho(K))$ can be extended to a morphism $K \rightarrow E(\rho(K))$. Now, $f(K) \subset E(\rho(K)) \cdot K$ since $K^{2}=K$. But, by hypothesis, $E(\rho(K))=$ $\rho(E(\rho(K))=E(\rho(K)) \cdot D$. Hence $f(K) \subset E(\rho(K)) \cdot D \cdot K=0$. Thus $\rho(K)=0$. As $\rho$ is hereditary, this means that $D \cap K=0$ which gives $R=D \oplus K$, a ring direct sum. From this it follows that $\rho$ is splitting by [6, Theorem 2.4].

COROllary 1.6. Let $R$ be a left 1-FGFP ring. Then the following are equivalent on any hereditary cotorsion radical $\rho$ on $\mathcal{M}_{R}$. (1). $\rho$ is splitting (2) $\rho(E(S))=0$ or $E(S)$ for any simple module $S$ in $\mathcal{M}_{R}$.

\section{Proof. Use (1.1), (1.4) and (1.5).}

PROPOSITION 1.7. Let $R$ be (i) a right self-injective cogenerator ring or (ii) a right or left order in a semi-simple Artin ring, then all hereditary cotorsion radicals for $\mathcal{M}_{R}$ are splitting.

Proof. (i) If $R$ is a right self-injective cogenerator ring, then by Utumi [13], there exist primitive orthogonal idempotents $e_{1}, \cdots, e_{n}$ in $R$ such that each $e_{\imath} R$ contains a unique simple submodule $S_{\imath}$ and a unique simple factor module $F_{t}=e_{t} R / e_{J} J$ [where $J$ is the Jacobson radical of $R$ ] such that $e_{i} R$ is the injective hull of $S_{t}$ and the projective cover of $F_{1}$ for each $i$. Further, the collections $\left\{S_{i}\right\}$ and $\left\{F_{1}\right\}$ each provide a complete set of representatives of the isomorphism classes of simple right $R$-modules so that we can assume $\left\{S_{l}\right\}=\left\{F_{i}\right\}$. Let $\rho\left(e_{\imath} R\right)=0$ for $i=1, \cdots, k$ and $\rho\left(e_{1} R\right) \neq 0$ for $i=k+1, \cdots, n$, for a hereditary cotorsion radical $\rho$ for 
$\mathcal{M}_{R}$. Then $\rho\left(S_{i}\right)=0=\rho\left(F_{1}\right)$ for $i=1, \cdots, k$ and $\rho\left(S_{\imath}\right) \neq 0$ for $i=$ $k+1, \cdots, n$. Hence $\left\{S_{1}, \cdots, S_{k}\right\}=\left\{F_{1}, \cdots, F_{k}\right\} \quad$ or equivalently $\left\{S_{k+1}, \cdots, S_{n}\right\}=\left\{F_{k+1}, \cdots, F_{n}\right\}$. Thus for $k+1 \leqq i \leqq n \rho\left(F_{i}\right) \neq 0$ so that $\rho\left(e_{i} R\right)=e_{i} R$ as otherwise $\rho\left(e_{i} R\right) \subset e_{i} J$ and hence there exists an epimorphism $e_{i} R / \rho\left(e_{i} R\right) \rightarrow e_{i} R / e_{i} J$ implying $\rho\left(e_{i} R / e_{i} J\right)=\rho\left(F_{i}\right)=0$. This shows that for each $i=1, \cdots, n, \quad \rho\left(e_{i} R\right)=\rho\left(E\left(S_{i}\right)\right)=0$ or $\rho\left(e_{i}(R)=E\left(S_{i}\right)\right.$. Hence the result follows from (1.6).

(ii) If $R$ is a right or left order in a semi-simple Artin ring, then $R$, being a subring of a left 1-FGFP ring, is itself a left 1-FGFP ring [Jondrup [7]]. Hence, if $\rho$ is a hereditary cotorsion radical for $\mathcal{M}_{R}$, then, by (1.1), $\rho(R)=\operatorname{Re}$ for an idempotent $e$ of $R$. It is easily shown that $e$ is a central idempotent, using the fact that $R$ has no nonzero nilpotent ideals. Hence $\rho$ is splitting, by (4) of (1.5).

On the splitting of cotorsion radicals in commutative rings, we have the following

Proposition 1.8. Let $R$ be a commutative ring. Then the following are equivalent on any cotorsion radical $\rho$ on $\mathcal{M}_{R}$.

(1) $\rho$ is splitting

(2) $\rho$ commutes with direct products

(3) $\rho(R)$ is finitely generated

If, further, each projective ideal in $R$ is finitely generated then the above are equivalent to

(4) $\rho$ is hereditary.

Proof. $1 \Rightarrow 2 \Rightarrow 3$ by (1.3.) $3 \Rightarrow 1$ follows from [6, Theorem 2.4] since $\rho(R)$ is idempotent and it is well known that any finitely generated idempotent ideal in a commutative ring is generated by an idempotent. The last part of the proposition follows from (1.1) and the fact that if each projective ideal of a commutative ring is finitely generated, then each cyclic flat module over $R$ is projective [Vasconcelos [14]].

REMARK $1 . \quad 1$ and 4 of the above proposition need not be equivalent in general. For instance, if $R$ is a commutative von Neumann regular ring which is not Artin semi-simple, then $R$ has a nonfinitely generated ideal $K$. If $\rho$ is the cotorsion radical defined by $\rho(M)=M K$ for every $R$-module $M$ then $\rho$ is a hereditary cotorsion radical that is not splitting. This example also shows that the hypothesis 'left 1-FGFP' cannot be dropped in Corollary (1.6). For any simple module over $R$ is injective and hence (2) of (1-6) is satisfied but (1) is not true for $\rho$.

REMARK 2. It seems worthwhile pointing out that the heredity of a cotorsion radical and the splitting of a hereditary cotorsion radical are 
completely determined by their behaviour with respect to $R_{R}$. For, a cotorsion radical $\rho$ for $\mathcal{M}_{R}$ is hereditary if and only if $\rho(M) \cap N=\rho(N)$ for all modules $M, N$ in $\mathcal{M}_{R}$ with $N \subset M$. However, by (1.1), it is enough to check this condition for $R_{R}$ since it is well known that, if $K$ is a left ideal of $R$, then, the left module $R / K$ is flat if and only if $A \cap K=A K$ for all right ideals $A$ of $R$. Thus $R_{R}$ is a test module for heredity. Similarly, if $\rho$ is a hereditary cotorsion radical for $\mathcal{M}_{R}$, then $R_{R}$ is a test module for the splitting of $\rho$. For, if $\rho(R)$ is a direct summand of $R_{R}$, i.e. $R=\rho(E) \oplus I$ for a right ideal $I$, then $R=\rho(R)+R I$ and by the heredity of $\rho, \rho(R) \cap R I=0$. Thus $\rho(R)$ is generated by a central idempotent and $\rho$ is splitting.

2. Cotorsion radicals for $\mathcal{M}_{R}$ and ${ }_{R} \mathcal{M}$. Any cotorsion radical $\rho$ for $\mathcal{M}_{R}$ induces a cotorsion radical $\rho^{\circ}$ on ${ }_{R} \mathcal{M}$ given by $\rho^{\circ}(M)=\rho(R) \cdot M$ for each $M$ in ${ }_{R} M$. The correspondence $\rho \rightarrow \rho^{\circ}$ is one-one between cotorsion radicals for $\mathcal{M}_{R}$ and the cotorsion radicals for ${ }_{R} \mathcal{M}$. It is clear that if $\rho$ splits, then $\rho^{\circ}$ is hereditary. Conversely, if $\rho^{\circ}$ splits, then $\rho=\left(\rho^{\circ}\right)^{\circ}$ is hereditary. The requirement that both $\rho$ and $\rho^{\circ}$ be hereditary can be quite strong, as the following proposition shows:

Proposition 2.1. Let $R$ be a right and left 1-FGFP ring and $\rho$ be a hereditary cotorsion radical for $\mathcal{M}_{R}$. Then $\rho$ splits if and only if $\rho^{\circ}$ is hereditary.

Proof. As $\rho$ is hereditary, $\rho(R)=\operatorname{Re}$ for an idempotent $e$. If $\rho^{\circ}$ is also hereditary, then $\rho(R)=f R$ for an idempotent $f$. Hence $\rho(R)$ is a ring direct summand of $R$ and hence $\rho$ splits. The converse follows by (1.1).

COROLLARY 2.2. Let $R$ be a right and left 1-FGFP ring. Then, for any cotorsion radical $\rho$ on $\mathcal{M}_{R}$, both $\rho$ and $\rho^{\circ}$ are hereditary if and only if both $\rho$ and $\rho^{\circ}$ split.

Apart from the above correspondence between arbitrary cotorsion radicals, a correspondence between the hereditary cotorsion radicals for $\mathcal{M}_{R}$ and those for ${ }_{R} \mathcal{M}$ can be obtained for a right and left 1-FGFP ring as follows.

Proposition 2.3. Let $R$ be a right and left 1-FGFP ring, then there exists a one-one correspondence between (i) hereditary cotorsion radicals for $\mathcal{M}_{R}$ (ii) hereditary cotorsion radicals for ${ }_{R} \mathcal{M}$ and (iii) idempotents $e$ of $R$ such that Re is an ideal. This correspondence is such that the corresponding hereditary cotorsion radicals split together and this happens if and only if the corresponding idempotent is central. 
Proof. If $\rho$ is a hereditary cotorsion radical for $\mathcal{M}_{R}$, then $\rho(R)=\mathrm{Re}$ for an idempotent $e$. Since $(1-e) R$ is an ideal which is also idempotent, $\rho^{*}(M)=(1-e) R \cdot M$ for each $M$ in ${ }_{R} M$ defines a hereditary cotorsion radical for ${ }_{R} M$. The correspondence $\rho \rightarrow \rho^{*}$ is clearly one-one between the hereditary cotorsion radicals for $\mathcal{M}_{R}$ and those for ${ }_{R} \mathcal{M}$. If $\rho$ splits, then $\rho(R)=f R$ for an idempotent $f$ which means that $e=f$ is a central idempotent, so that $(1-e)$ is also a central idempotent and hence $\rho^{*}(R)$ splits.

COROllary 2.4. Let $R$ be a right and left 1-FGFP ring. If all hereditary cotorsion radicals for $\mathcal{M}_{R}$ split, then, for any idempotent $e$ of $R$, $R e$ is an ideal if and only if $e$ is a central idempotent.

Remark. In view of (i) of (1.7), this Corollary generalizes Proposition (4.4) of Anderson [1] where the same conclusion is derived for quasi-Frobenius rings.

\section{Cotorsion radicals induced by projective modules.} Let $P$ be a projective right $R$-module. Beachy [3] defined for any $M$ in $M_{R}, \rho_{P}(M)=\operatorname{Tr}_{M}(P)=$ the sum of all the homomorphic images of $P$ in $M$, and showed that $\rho_{P}$ is a cotorsion radical for $\mathcal{M}_{R}$ with $\rho_{P}(R)=\operatorname{Tr}_{R}(P)=$ trace ideal of $P$ in $R$. Beachy [3, Theorem 1.8] proved that if every idempotent ideal of $R$ has a projective cover, all cotorsion radicals for $M_{R}$ are of the form $\rho_{P}$ for projective modules $P$. Right perfect rings and semiperfect right Noetherian rings are examples of such rings. We now point out another class of rings with this property. A ring $R$ is said to be right (left) $\boldsymbol{N}_{0}$-hereditary if every countably generated right (left) ideal of $R$ is projective.

Proposition 3.1. If $R$ is a right and left $\boldsymbol{\aleph}_{0}$-hereditary ring (in particular if $R$ is von Neumann regular), then each cotorsion radical for $\mathcal{M}_{R}$ is of the form $\rho_{P}$ for a projective module $P$ in $\mathcal{M}_{R}$.

Proof. By Corollary 5.3 of Bergman [4], each idempotent ideal of such a ring is the trace ideal of a projective module. Hence if $\rho$ is a cotorsion radical for $\mathcal{M}_{R}$, then $\rho(R)=\operatorname{Tr}_{P}(R)$ for some projective right module $P$, so that, for any module $M$ in $\mathcal{M}_{R}, \rho(M)=M \cdot \rho(R)=$ $M \cdot \operatorname{Tr}_{R}(P)=\operatorname{Tr}_{M}(P)=\rho_{P}(M)$. Thus $\rho=\rho_{P}$.

We indicate now some situations where $\rho_{P}$ is hereditary. It is clear from (1.1) that, if $R$ is a left 1-FGFP ring, then $\rho_{P}$ is hereditary for a projective right module $P$ if and only if $\operatorname{Tr}_{R}(P)=\operatorname{Re}$ for an idempotent $e$ and that if $R$ is a von Neumann regular ring, then, for every projective module $P, \rho_{P}$ is hereditary. We give a generalization of the latter fact now. A right $R$-module $P$ has been called regular if $P$ is projective and every homomorphic image of $P$ is flat [Ware [15]]. 
Proposition 3.2. If $P$ is a regular module, then $\rho_{P}$ is hereditary.

Proof. It suffices to show that, for each $a$ in $T\left(=\operatorname{Tr}_{R}(P)\right)$, there is $x$ in $T$ such that $a=a x$. For this implies that $R / T$ is left $R$-flat and hence (1.1) applies. So let $a \in T$. Then $a=\sum_{1}^{k} f_{t}\left(p_{t}\right)$ with $f_{i} \in \operatorname{Hom}(P, R)$ and $p_{i} \in P$. As $P$ is regular, by Theorem 2.3 of Zelmanowitz [16], for every $p$ in $P$, there is $t$ in $T$ such that $p=p t$. Thus there is $t_{1}$ in $T$ with $p_{1}=p_{1} t_{1}$ and $t_{2}$ in $T$ with $p_{2}-p_{2} t_{1}=$ $\left(p_{2}-p_{2} t_{1}\right) t_{2}$. Hence $p_{2}=p_{2}\left(t_{1}+t_{2}-t_{1} t_{2}\right)$ and clearly $p_{1}=p_{1}\left(t_{1}+t_{2}-t_{1} t_{2}\right)$ and $t_{1}+t_{2}-t_{1} t_{2} \in T$. Proceeding thus, we get $x \in T$ with $p_{i}=p_{i} x$ for $i=1,2,3, \cdots, k$. Evidently $a=a x$.

Proposition 3.3. Let $R$ be a right nonsingular ring (i.e. a ring with zero right singular ideal), and $P$ be a projective right $R$-module which is also injective. Then the following are equivalent (1) $P$ is a regular module (2) $\rho_{P}$ is hereditary (3) $\operatorname{Tr}_{R}(P)$ is a von Neumann regular ring (not necessarily with identity).

Proof. $1 \Rightarrow 2$ : follows from Proposition 3.2.

$2 \Rightarrow 3$ : Let $T=\operatorname{Tr}_{R}(P)$ and $a \in T$.

Then $a R=\sum_{f_{i} \in \operatorname{Hom}(P, a R)} f_{i}(P)$ as $\rho_{P}$ is hereditary. Hence there exists an epimorphism from a direct sum $Q$ of a finite number of copies of $P$, to $a R$. As $Q$ is injective and $a R$ is nonsingular, the kernel of this epimorphism has no proper essential extensions and hence is a direct summand of $Q$. In other words, $a R$ is injective and so $a R=e R$ for an idempotent $e$. Since $a R=a T$, this gives $a=a x a$ for $x \in T$.

$3 \Rightarrow 1$ : Let $I$ be the collection of all idempotents in $\operatorname{Tr}_{R}(P)$. Then $\operatorname{Tr}_{R}(P)=\sum_{e_{\alpha} \in I} \operatorname{Re}_{\alpha} R=\Sigma_{I} \operatorname{Tr}_{R}\left(e_{\alpha} R\right)=\operatorname{Tr}_{R}\left(\Sigma_{I} \oplus e_{\alpha} R\right)=\operatorname{Tr}_{R}(Q) \quad$ where $Q=\Sigma_{I} \oplus e_{\alpha} R$. Hence $P$ is a homomorphic image of a direct sum of copies of $Q$ and as $Q$ is a direct sum of the regular modules $e_{\alpha} R$ and hence a regular module, so is $P$.

EXAMPLE. Let $R$ be the ring of $2 \times 2$ lower triangular matrices over a field $F$ and let $e=\left[\begin{array}{ll}0 & 0 \\ 0 & 1\end{array}\right] . \quad R$ is a hereditary ring, $e R$ is a finitely generated, faithful projective module which is also injective and $\operatorname{Tr}_{R}(e R)=e R$. Since $\rho_{e R}$ commutes with direct products but $e$ is not a central idempotent it follows by (1.4), that $\rho_{e R}$ is not hereditary.

For the rest of this section, we fix the following notation: $P$ is a finitely generated projective right $R$-module, $T=\operatorname{Tr}_{R}(P), S=$ End $\left(P_{R}\right)$ and $P^{*}=\operatorname{Hom}_{R}(P, R)$. It is known that $P^{*}$ is a finitely generated, projective left $R$-module with $T=\operatorname{Tr}_{R}\left(P^{*}\right)$ and $S=\operatorname{End}_{R}\left(P^{*}\right)$. If $\rho=\rho_{P}$, 
then ${ }_{p *} \rho=\rho^{\circ}$ in the notation of the previous section. Let $F$ be the functor $P \otimes_{R}(-):{ }_{R} \mathcal{M} \rightarrow{ }_{s} \mathcal{M}$. Morita has shown that if $P$ is a finitely generated projective genrator, then $F$ is a category equivalence, preserving in particular, projective covers and injective envelopes. In [1], Anderson considered the question: if $P$ were not necessarily a generator, then under what conditions on $P$, will $F$ preserve projective covers and injective envelopes? This question is now shown to be related to the heredity of the cotorsion radicals $\rho$ and $\rho^{\circ}$.

Proposition 3.5. F preserves injective envelopes if and only $\rho^{\circ}$ is hereditary.

Proof. By Theorem 2.4 of Anderson [1], $F$ preserves injective envelopes if and only if $x \in T x$ for every $x \in T$. The latter condition is equivalent to: $R / T$ is right $R$-flat, which is, in turn, equivalent to: $\rho^{\circ}$ is hereditary, by the mirror dual of (1.1).

COROLlary 3.6. Let $P$ be a regular and injective module. Then $S$ is a self-injective, von Neumann regular ring.

Proof. By (3.2), $\rho$ is hereditary. Hence, by the mirror dual of (3.5) the functor $(-) \bigotimes_{R} P^{*}: M_{R} \rightarrow M_{S}$ preserves injectives. As $P$ is injective, this shows that $S \simeq P \otimes_{R} P^{*}$ is is self-injective. $\quad S$ is von Neumann regular, by Theorem 3.6 of Ware [15].

\section{REMARK. This extends Corollary (2.6) of Anderson [1].}

We close with a remark on the connection between torsion radicals and flat modules. Let us say that a torsion radical $\rho$ on $\mathcal{M}_{R}$ is given by a flat module if there is a flat left $R$-module $Q$ such that, for any $M \in \mathcal{M}_{R}$, $\rho(M)=M$ if and only if $M \otimes_{R} Q=0$. Torsion radicals of this type and their associated quotient rings were studied by Morita in [9]. In [11] Shelter and Roberts considered the question: which torsion radicals are given by flat modules? They have shown that: (i) If $R$ is any ring and $\rho$ a torsion radical on $M_{R}$ such that the Gabriel filter associated with $\rho$ has a countable base, then $\rho$ is given by a flat module. (ii) If $R$ is a commutative Noetherian ring, then every torsion radical on $\mathcal{M}_{R}$ is given by a flat module. In this connection, we have

Proposition 3.7. (i) If $R$ is any ring and $\rho$ is a hereditary cotorsion radical on $\mathcal{M}_{R}$, then $\rho$ is given by a flat module. (ii) If $R$ is a primary decomposable ring, then every torsion radical on $\mathcal{M}_{R}$ is given by a flat module. 
Proof. (i) If $\rho$ is a cotorsion radical on $\mathcal{M}_{R}$, then $\rho(M)=M \Leftrightarrow$ $M \otimes_{R}(R / \rho(R))=0$ because $\rho(M)=M \cdot \rho(R)$ and $M \otimes_{R}(R / \rho(R)) \simeq$ $M / M \cdot \rho(R)$. If $\rho$ is hereditary, then $R / \rho(R)$ is left $R$-flat. So $\rho$ is given by the flat module $R / \rho(R)$.

(ii) If $R$ were a primary decomposable ring, then, by Teply [12, Theorem 3.1 and 3.3] every torsion radical on $\mathcal{M}_{R}$ is a hereditary cotorsion radical. Hence, by (i) every torsion radical on $\mathcal{M}_{R}$ is given by a flat module.

\section{REFERENCES}

1. F. W. Anderson, Endomorphism rings of projective modules, Math. Z., 111 (1969), 322-332.

2. G. Azumaya, Some properties of TTF classes, Proc. Conf. on Orders, Group Rings, and Related Topics, Lecture Notes in Math., vol. 353, Springer-Verlag, Berlin, 1973.

3. J. A. Beachy, Cotorsion radicals and projective modules, Bull. Austral. Math. Soc., 5 (1971), 241-253.

4. G. M. Bergman, Infinite multiplication of ideals, J. Algebra, 24 (1973), 56-70.

5. S. U. Chase, Direct products of modules, Trans. Amer. Math. Soc., 97 (1960), 457-473.

6. J. P. Jans, Some aspects of torsion, Pacific J. Math., 15 (1965), 1249-1259.

7. S. Jondrup, On finitely generated flat modules $\Pi$, Math. Scand., 27 (1970), 105-112.

8. J. M. Maranda, Injective structures, Trans. Amer. Math. Soc., 110 (1964), 98-135.

9. K. Morita, Flat modules, injective modules and quotient rings, Math. Z., 120 (1972), 25-40.

10. V. S. Ramamurthi, On splitting cotorsion radicals, Proc. Amer. Math. Soc., 39 (1973), 457-461.

11. W. Shelter and P. Roberts, Flat modules and torsion theories, Math. Z., 129 (1972), 331-334.

12. M. L. Teply, Homological dimension and splitting torsion theories, Pacific J. Math., 34 (1970), 193-205.

13. Y. Utumi, Self-injective rings, J. Algebra, 6 (1967), 56-64.

14. W. V. Vasconcelos, On projective modules of finite rank, Proc. Amer. Math. Soc., 22 (1969), 430-433.

15. R. Ware, Endomorphism rings of projective modules, Trans. Amer. Math. Soc., 155 (1971), 233-256.

16. J. Zelmanowitz, Regular modules, Trans. Amer. Math. Soc., 163 (1972), 341-355.

Received February 10, 1975 and in revised form October 29, 1975.

De la Salle College

AND

UNIVERSITY OF KANSAS 



\section{Pacific Journal of Mathematics}

\section{Vol. 62, No. $1 \quad$ January, 1976}

Mieczyslaw Altman, Contractor directions, directional contractors and

directional contractions for solving equations . .................. 1

Michael Peter Anderson, Subgroups of finite index in profinite groups .........

Zvi Arad, Abelian and nilpotent subgroups of maximal order of groups of odd order

John David Baildon and Ruth Silverman, On starshaped sets and Helly-type theorems ..........................................

John W. Baker and R. C. Lacher, Some mappings which do not admit an

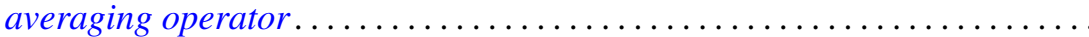

Joseph Barback, Composite numbers and prime regressive isols . . . . . . . . . .

David M. Boyd, Composition operators on $H^{p}(A) \ldots \ldots \ldots \ldots \ldots \ldots \ldots$

Maurice Chacron, Co-radical extension of PI rings . . . . . . . . . . . . .

Fred D. Crary, Some new engulfing theorems . . . . . . . . . . . . . . .

Victor Dannon and Dany Leviatan, A representation theorem for convolution transform with determining function in $L^{p} \ldots \ldots \ldots \ldots \ldots \ldots \ldots \ldots \ldots \ldots \ldots \ldots \ldots \ldots$

Mahlon M. Day, Lumpy subsets in left-amenable locally compact

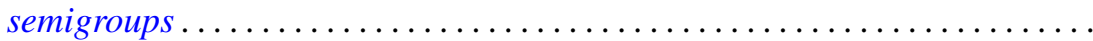

Michael A. Gauger, Some remarks on the center of the universal enveloping algebra of a classical simple Lie algebra . .

David K. Haley, Equational compactness and compact topologies in rings

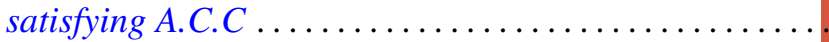

Raymond Heitmann, Generating ideals in Prüfer domains .

Gerald Norman Hile, Entire solutions of linear elliptic equations with

Laplacian principal part. .

Richard Oscar Hill, Moore-Postnikov towers for fibrations in which $\pi_{1}$ (fiber) is non-abelian

John Rast Hubbard, Approximation of compact homogeneous maps . .

Russell L. Merris, Relations among generalized matrix functions . .

V. S. Ramamurthi and Edgar Andrews Rutter, On cotorsion radicals ...

Ralph Tyrrell Rockafellar and Roger Jean-Baptiste Robert Wets, Stochastic

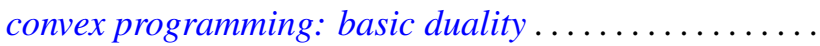

Alban J. Roques, Local evolution systems in general Banach spaces ..

I. Bert Russak, An indirect sufficiency proof for problems with bounded state variables.

Richard Alexander Sanerib, Jr., Ultrafilters and the basis property. .

H. A. Seid, The decomposition of multiplication operators on $L_{p}$-spaces . .

Franklin D. Tall, The density topology .................. 